\title{
Solid electrolyte interphase in water-in-salt electrolytes
}

\author{
Dezhong Liu, Lixia Yuan ${ }^{*}$ and Yunhui Huang
}

\begin{abstract}
The water-in-salt strategy successfully expands the electrochemical window of the aqueous electrolyte from 1.23 to $\sim 3.0 \mathrm{~V}$, which can lead to a breakthrough in the energy output of the aqueous battery system while maintaining the advantage of high safety. The expanded electrochemical window of the water-in-salt electrolytes can be ascribed to the decreased water activity and the solid electrolyte interphase formed on the anode. The solid electrolyte interphase in the aqueous system is not fully understood, and the basic composition, the structure, and the formation mechanism are still cloaked in mystery. This perspective summarizes the published research with emphasis on the most possible formation mechanism and composition of the interphase layer in the aqueous system. Further understanding of the interphase as well as rounded assessment of the water-in-salt electrolyte in practical operating conditions is encouraged. The full understanding of the interface will guide the design of aqueous electrolytes and help to build novel aqueous batteries with high safety and high energy density.
\end{abstract}

Keywords: solid electrolyte interphase, water-in-salt electrolytes, aqueous rechargeable $\mathrm{Li}$-ion batteries

\section{INTRODUCTION}

As rechargeable Li-ion batteries (LIBs) serve the modern industry and society ubiquitously, public concerns over the safety of LIBs are increasing, especially in large-scale applications such as electric vehicles (EVs). Current LIBs adopt flammable organic solvent electrolytes, which play a critical role in the combustion or explosion accidents caused by LIBs in recent years. Therefore, the newly emerged LIB system based on aqueous electrolytes shows huge appeal. Replacing organic electrolytes with aqueous electrolytes can offer not only high safety but also low cost, environmental benignity, and high tolerance against abnormal abuse [1,2]. The batteries with solid-state electrolytes also have a great advantage in safety, but they require a particular manufacturing process, which poses a big challenge for the battery industry. In contrast, aqueous LIBs (ALIBs) can be perfectly compatible with the current LIB assembly line and show promise for future large-scale production.

In 1994, the first ALIB based on rocking-chair battery chemistry was proposed by $\mathrm{Li}$ et al. [3], with $\mathrm{LiMnO}_{4}$ as the cathode, $\mathrm{VO}_{2}$ as the anode, and a solution with $5 \mathrm{~mol} \mathrm{~L}^{-1} \mathrm{LiNO}_{3}$ in water as the electrolyte. The cell achieves an energy density of $\sim 55 \mathrm{~W} \mathrm{~h} \mathrm{~kg}^{-1}$ with an average discharge voltage of $\sim 1.5 \mathrm{~V}$, but the cycle stability is poor. Since then, by optimizing the electrodes, the electrolytes, and the current collectors, great progress has been made to improve the specific capacity, cycling stability, and rate capability [4]. However, the narrow electrochemical stability window (ESW) of water $(1.23 \mathrm{~V})$ limits the operating voltage (usually lower than $1.5 \mathrm{~V}$ ) of ALIBs. The ESW of water rules out most of the energydense anodes and cathodes, which always work beyond the voltage range, otherwise the water splitting reactions would dominate the electrode reaction, inhibiting the energy-bearing lithiation/delithiation process [5]. The organic LIB system with ethylene carbonate (EC) as cosolvent for the electrolytes also encounters a similar issue; the insertion potential of lithium ions in graphite, which is the most widely used anode material of LIBs, is beyond the thermodynamic ESW of EC. During the initial lithiation process, the EC molecules are reduced, and the products form a passive layer deposited on the surface of the graphite anode, which is the well-known "solid electrolyte interphase" (SEI) in LIBs. The SEI successfully prevents the further decomposition of electrolytes, thus enabling the subsequent charging-discharging reaction of the battery [6,7]. The passive layer was first observed on a lithium metal anode soaked in a propylene carbonate

State Key Laboratory of Materials Processing and Die \& Mould Technology, School of Materials Science and Engineering, Huazhong University of Science and Technology, Wuhan 430074, China

* Corresponding author (email: yuanlixia@hust.edu.cn) 
(PC) electrolyte by Dey et al. [8] in 1970. Then it was named the SEI by Peled [9] in 1979. The SEI widely exists on the surface of vast majority of the anodes of LIBs and also on some cathodes (the passive layer on cathode surface is more often referred to as the "cathode electrolyte interphase" (CEI)). The SEI is a solid-state electrolyte that can insulate electronics while conducting the lithium ions. For the current LIBs, the SEI plays an important role in stabilizing the electrode structure and suppressing the side reactions during the repeated charging-discharging cycles. As the SEI passivates the anodes efficiently, the LIBs can operate in an expanded voltage range successfully. The breakthrough of LIBs in energy density, to a large extent, is rooted in the high voltage output of the battery.

Unlike the organic LIB system, an efficient protective SEI cannot form on the aqueous counterpart, as the splitting reaction products of water are gases $\left(\mathrm{O}_{2}\right.$ and $\left.\mathrm{H}_{2}\right)$, making it difficult to expand the ESW of aqueous electrolytes via the SEI strategy. Recently, a groundbreaking work by Suo et al. [10] proposed a new aqueous electrolyte system, the "water-in-salt" electrolyte (WiSE), which is an aqueous solution with high salt concentration of $21 \mathrm{~mol} \mathrm{~kg}^{-1}$ lithium bis(trifluoromethanesulfonyl) imide (LiTFSI). The WiSE successfully expands the ESW of the aqueous electrolyte from $\sim 1.23$ to $>3.0 \mathrm{~V}$. Later studies demonstrate that other lithium salts or bisalts based on fluoroalkyl sulfonylimide anions or fluoroalkyl sulfonate anions, e.g., lithium bis(pentafluoroethanesulfonyl)imide (LiBETI) and lithium trifluoromethane sulfonate (LiOTf), could also largely expand the ESW with a super-concentrated solution [1113]. With a widened ESW, the energy-dense anode/ cathode materials with lower/higher redox voltage, such as $\mathrm{Li}_{4} \mathrm{Ti}_{5} \mathrm{O}_{12}$ and $\mathrm{LiNi}_{0.5} \mathrm{Mn}_{1.5} \mathrm{O}_{4}$, which are excluded from the aqueous system, can be used to construct ALIBs, resulting in significantly improved voltage output $(2.1 \mathrm{~V})$ as well as energy density $\left(100 \mathrm{~W} \mathrm{~h} \mathrm{~kg}^{-1}\right)$. The widened ESWs of WiSEs can be mainly ascribed to the reduced water activity due to the significantly decreased concentration of the free water molecules, and the SEI formed on the anode surface also plays an important role. Although not all ESWs of WiSEs are expanded by the SEI, as for the cathodic limit, the protective interphase in the anode significantly contributes to the delay of the hydrogen evolution reaction (HER) and therefore broadens the cathodic limit $[14,15]$. However, so far, a comprehensive understanding of the SEI in WiSEs has not been reached in terms of the formation process, the interphase chemistry, and physical properties. In some respects, the SEI in
WiSEs can be more elusive than that in organic electrolytes due to its continuous dissolution/formation in aqueous electrolytes and more side reactions that compete with formation reactions [16]. Efforts are urgently needed to unveil the mystery of the SEI in the aqueous system, which will provide guidelines to tailor the electrolyte composition and therefore further improve the electrochemical performances of the aqueous batteries.

\section{CURRENT STATUS}

\section{ESW expanding mechanisms of WiSEs}

The WiSE concept greatly diversifies the battery chemistry. In addition to ALIBs, it also proves feasibility in conversion reactions (e.g., sulfur) [17] and other metal ion intercalation systems (e.g., $\mathrm{Na}^{+}$and $\mathrm{K}^{+}$) [18-20]. The WiSE concept has also been applied in supercapacitors $[21,22]$. In turn, the versatility further evidences the effectiveness of expanding the ESW with the WiSE concept. While WiSE improves the oxidative and reductive stability of the aqueous solution simultaneously, there is usually an uneven position of cathodic and anodic limits, which stems from the preferential distribution of water molecules and salt anions at the inner Helmholtz interface of the electrode as potential is applied $[23,24]$. In the cathode side, anions tend to accumulate on the cathode surface upon positive polarization and exclude water from the inner Helmholtz layer, largely decreasing the water activity [24]. The kinetic barrier for water molecules to free themselves from chain-like aggregation, as proposed Zheng et al. [25], is mainly responsible for the expansion of the anodic limit. As shown in Fig.1a, the aqueous solution with concentrated $\mathrm{LiNO}_{3}$ (the salt/water molar ratio is 1:2.5) shows an oxidation potential much higher than the dilute counterpart [25]. Another WiSE with a highly concentrated eutectic mixture of lithium and potassium acetate also achieves an expanded oxidation limit [26]. Therefore, the oxidative window is largely widened without obvious SEI formation, while for the anode side, the broadening mechanism is quite different. When the anode is negatively polarized, anions tend to be expelled from the anode surface, and cations solvated by water molecules are attracted, making the HER more likely to occur. Fig. 1b, c show the linear sweep voltammetry of various electrolytes with different salts and concentrations [27]. Although the glassy carbon (GC) and platinum disk electrodes have different catalytic abilities for the HER, it can still be concluded that the onset reduction potential is independent of the anion concentration. As the potential further moves to the ne- 

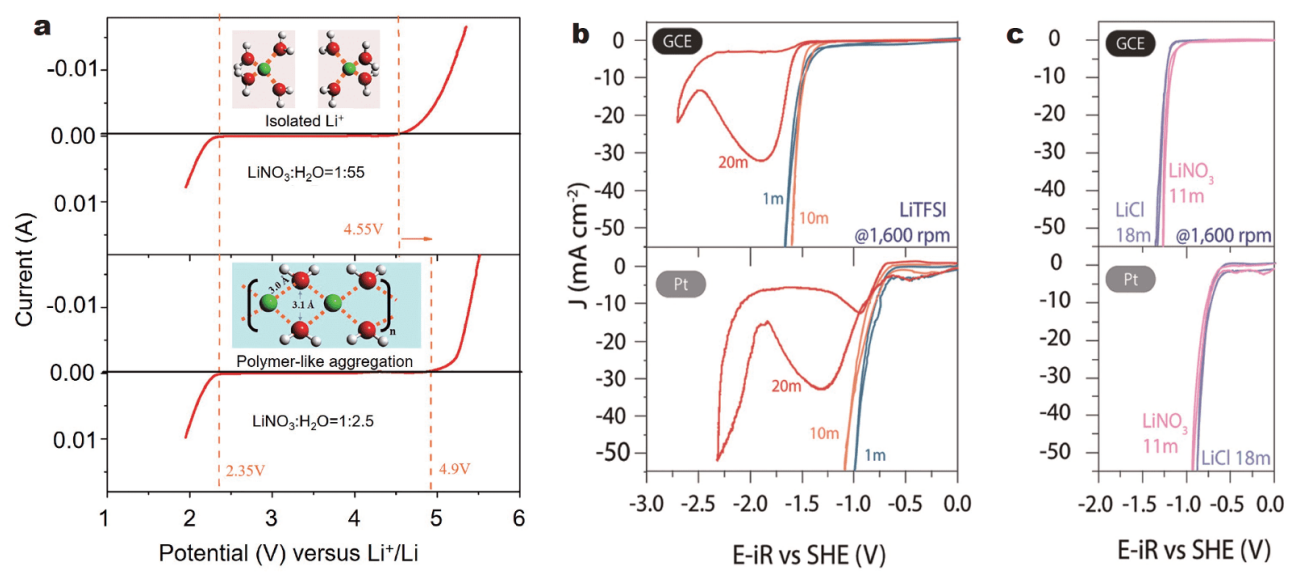

Figure 1 (a) Linear sweep voltammetry of the dilute $\mathrm{LiNO}_{3}$ aqueous solution (salt/water molar ratio is 1:55) and highly concentrated solution (1:2.5). Reproduced with permission from Ref. [25], Copyright 2018, Elsevier. Cyclic voltammograms of (b) LiTFSI aqueous electrolytes with various concentrations on GC (top) and platinum (bottom) disk electrodes and (c) the same rotating disk experiment performed in $\mathrm{LiNO}_{3}$-saturated solution $\left(\sim 11 \mathrm{~mol} \mathrm{~kg}^{-1}\right.$, pink) and $\mathrm{LiCl}$ saturated solution $\left(\sim 18 \mathrm{~mol} \mathrm{~kg}^{-1}\right.$, purple). Reproduced with permission from Ref. [27], Copyright 2018, Royal Society of Chemistry. The " $m$ " after the numbers in $(b, c)$ stands for a unit of $\mathrm{mol} \mathrm{kg}^{-1}$.

gative side, a cathodic peak appears in the LiTFSI-based WiSE, which corresponds to the SEI formation reactions; the solid products of TFSI ${ }^{-}$decomposition construct the passive layer. The $\mathrm{TFSI}^{-}$-derived SEI delays the potential of the drastic HER current successfully. In contrast, the reduction peak is not observed in the inorganic salt-based WiSEs, which thus show an inferior cathodic stability. Obviously, the passivation from an ionic conductive and electrically insulated SEI plays the key role in increasing the reductive stability of WiSEs [10,12]. The different widening mechanisms between cathode and anode make the expanded ESW always "positively biased" [23], and the "cathodic stability" poses the major challenge for the application of the WiSEs.

\section{The formation of SEI in WiSEs}

Several attempts have been made to verify the ingredients of the SEI and to speculate the formation process in the aqueous system. Since the primary difference between the dilute solutions and WiSEs is the concentration of lithium salt, some relation should exist between the SEI formation and the solvation structure of WiSEs. In the superconcentrated electrolytes, water molecules are insufficient to form the typical solvation structure; hence, anions participate in the primary solvation sheathing of $\mathrm{Li}^{+}$and therefore provide a high probability of the effective reduction of TFSI ${ }^{-}$. Here, it is believed that the fluorinated bulky anions (e.g., $\mathrm{TFSI}^{-}, \mathrm{BETI}^{-}$, and $\mathrm{OTf}^{-}$) are the active ingredients to form an effective SEI in WiSEs. For the fluorinated salt-based WiSEs, fluorides, including LiF and $\mathrm{CF}_{x}$ species, are usually found in the SEI layer on the cycled anode. According to the density functional theory (DFT) calculation, as the salt concentration increases, both the conduction band minimum of TFSI and the valence band maximum of water shift to a lower potential, resulting in premature TFSI $^{-}$reduction and delayed water oxidation [10]. The TFSI $^{-}$in aggregates such as $\mathrm{Li}_{2}(\mathrm{TFSI})\left(\mathrm{H}_{2} \mathrm{O}\right)_{x}$ can be reduced below $2.9 \mathrm{~V}$ vs. $\mathrm{Li}^{+} / \mathrm{Li}$, which is much higher than the reduction potential for isolated TFSI ${ }^{-}$at $1.4 \mathrm{~V}$ and HER at $2.63 \mathrm{~V}$.

Suo et al. $[10,16]$ have proposed that the reduction of TFSI $^{-}$can generate LiF, which stands as the main component of an SEI, and the reductions of dissolved $\mathrm{CO}_{2}$ and $\mathrm{O}_{2}$ yield $\mathrm{Li}_{2} \mathrm{CO}_{3}$ and $\mathrm{Li}_{2} \mathrm{O}$ in an SEI as well. The possible reactions are shown in Fig. 2a. Dubouis et al. [27] raised a different point. They found that the nucleophilic reaction between TFSI $^{-}$and $\mathrm{OH}^{-}$in the HER is more favored than the direct reduction of $\mathrm{TFSI}^{-}$. As shown in Fig. $2 b$, there is no evident reduction peak in a dry solution of $1 \mathrm{~mol} \mathrm{~L}^{-1}$ LiTFSI in [1-ethyl-3-methylImidazolium (Emim)][TFSI] in which water molecules are absent. In contrast, the reduction peak is prominent in the presence of water. The ${ }^{19} \mathrm{~F}$ nuclear magnetic resonance (NMR) spectrum (Fig. 2c) evidences that KF forms after heating KTFSI and $\mathrm{KOH}$, indicating that $\mathrm{TFSI}^{-}$is unstable under strong alkaline conditions such as the ones encountered during the initial HER at the negative electrode in WiSEs. Noticeably, in a non-fluorinated WiSE with $17 \mathrm{~mol} \mathrm{~kg}^{-1} \mathrm{NaClO}_{4}$ as salt, an SEI is also found with $\mathrm{Na}_{2} \mathrm{CO}_{3}$ and $\mathrm{NaOH}$ as the main ingredients [28]. The voltage decay curves and the transmission electron microscopy (TEM) image shown in Fig. $2 \mathrm{~d}$ indicate that a 

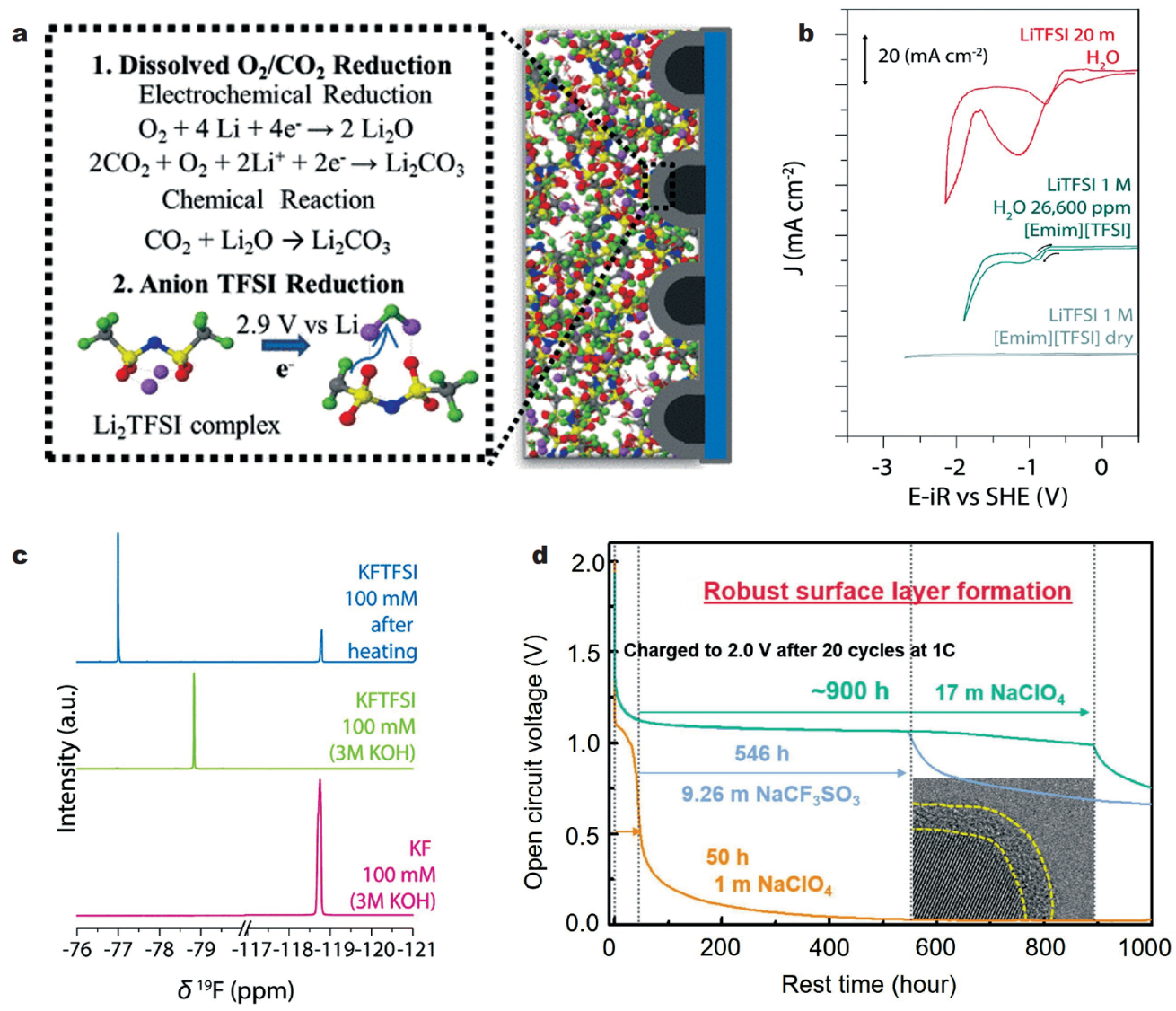

Figure 2 (a) SEI formation mechanisms in WiSE. Reproduced with permission from Ref. [16], Copyright 2017, American Chemical Society. (b) Cyclic voltammograms of the WISEs: $20 \mathrm{~mol} \mathrm{~kg}^{-1}$ LiTFSI in $\mathrm{H}_{2} \mathrm{O}$ (red), $1 \mathrm{~mol} \mathrm{~L}^{-1}$ LiTFSI in $\mathrm{H}_{2} \mathrm{O}$ saturated [Emim][TFSI] (blue), and $1 \mathrm{~mol} \mathrm{~L}{ }^{-1}$ LiTFSI in dry [Emim][TFSI] (gray). (c) ${ }^{19} \mathrm{~F}$ NMR analysis of the $100 \mathrm{mmol} \mathrm{L}^{-1} \mathrm{KTFSI}$ in $3 \mathrm{~mol} \mathrm{~L}^{-1} \mathrm{KOH}$ solution before (green) and after $60 \mathrm{~h}$ at $120^{\circ} \mathrm{C}$ (blue) compared with the same analysis replacing KTFSI by KF (pink). Reproduced with permission from Ref. [27], Copyright 2018, Royal Society of Chemistry. (d) Voltage decay curve of a $\mathrm{Na}_{4} \mathrm{Fe}_{3}\left(\mathrm{PO}_{4}\right)_{2}\left(\mathrm{P}_{2} \mathrm{O}_{7}\right) / \mathrm{NaTi}_{2}\left(\mathrm{PO}_{4}\right)_{3}$ full-cell at $100 \%$ state of charge (SOC) measured at $25^{\circ} \mathrm{C}$ in different aqueous electrolytes and TEM image of the $\mathrm{NaTi}_{2}\left(\mathrm{PO}_{4}\right)_{3}$ anode reconverted from $17 \mathrm{~mol} \mathrm{~kg}^{-1} \mathrm{NaClO}_{4}$ electrolyte. Reproduced with permission from Ref. [28], Copyright 2019, Elsevier.

robust surface layer forms on the anode after cycling in $17 \mathrm{~mol} \mathrm{~kg}^{-1} \mathrm{NaClO}_{4}$ solution. Apparently, here the SEI does not form through the reduction of $\mathrm{ClO}_{4}^{-}$; instead, the dissolved $\mathrm{CO}_{2}$ and $\mathrm{O}_{2}$ in electrolytes are responsible for the SEI formation. The formation route can also be supported by the existence of $\mathrm{Li}_{2} \mathrm{CO}_{3}$ and $\mathrm{LiOH}$ in the SEI of LIBs [16]. In this regard, fluorinated salt may be dispensable in the SEI formation. Taking all this evidence into consideration, the SEI in WiSEs is most likely a product blend of reactions involving anions of lithium salts, $\mathrm{CO}_{2}, \mathrm{O}_{2}$, and $\mathrm{OH}^{-}$, and the corresponding components include fluorides (such as metal fluorides and $\mathrm{CF}_{x}$ groups), carbonates, metal oxides, and metal hydroxides.

In carbonate-based organic electrolytes (e.g., $\mathrm{LiPF}_{6}$ in EC/PC), the main components of the SEI are decomposed products from both solvents and salts. These components include lithium alkylcarbonate, $\mathrm{Li}_{2} \mathrm{CO}_{3}$, and $\mathrm{LiF}[7,29]$. In contrast, in WiSEs, the only origination of the SEI is the electrolyte salts. Up to now, most of the reported WiSEs use fluorinated salts; as a result, fluoride species including $\mathrm{LiF}$ and $\mathrm{CF}_{x}$ are always the main species in the interphases. It is worth investigating whether this fluoridedominated SEI is the real SEI in practical cells because the post-mortem observations cannot perfectly represent the real SEI due to its high sensitivity to the ambient condition and the characterization tools [30]. Although LiTFSI can generate $\mathrm{LiF}$ under X-ray radiation and/or $\mathrm{Ar}^{+}$ etching [11], fluoride species are still considered as the most likely components of the SEI in fluorinated WiSEs, in view of the widely observed fluorides on the cycled electrode after rinsing, which has been evidenced by TEM, time-of-flight secondary ion mass spectrometry 
(TOF-SIMS), and solid-state NMR [10,16,27]. Moreover, among the reported interphase ingredients, $\mathrm{LiF}$ has the lowest solubility, making it more prone to depositing on the electrode surface instead of dissolving into the electrolyte.

Another question is whether the LiF-based SEI is the most suitable choice for aqueous batteries, as $\mathrm{LiF}$ itself is believed to be not only a good electronic insulator but also a poor ionic conductor $\left(\sim 10^{-31} \mathrm{~S} \mathrm{~cm}^{-1}\right)$ [31]. Actually, the SEI in WiSEs is more likely a mixture of $\mathrm{LiF}, \mathrm{Li}_{2} \mathrm{CO}_{3}$, and $\mathrm{LiOH}$. Zhang et al. [32] have found that the contact between $\mathrm{Li}_{2} \mathrm{CO}_{3}$ and $\mathrm{LiF}$ can boost the lithium ion transport and reduce the electron leakage simultaneously. This result sheds light on the ion transport mystery of the SEI, indicating that the fluorinated SEI is mixed with $\mathrm{Li}_{2} \mathrm{CO}_{3}$, which is rich in defects and disorders and can be more favorable in terms of the transport properties.

To further improve the stability of the aqueous electrolytes, some co-solvents and co-salts have also been developed. Among them, hybridizing aqueous and nonaqueous solvents can bring additional organic interphase ingredients into the SEI to further broaden the ESW [3335]. By analogy with the WiSE system, these electrolytes can be called "bisolvent-in-salt" hybrid electrolytes, where "bisolvent" stands for water and non-aqueous solvent. For instance, dimethyl carbonate (DMC) and acetonitrile (AN) have been applied as co-solvents, and organic species, carbonyl $(\mathrm{C}=\mathrm{O})$ and ethereal species $(\mathrm{C}-\mathrm{O})$ (from $\mathrm{DMC}$ ), nitrile $(\mathrm{C} \equiv \mathrm{N})$, and sulfamide (R-S-N-S) (from $\mathrm{AN})$ appear as the new interphase ingredients correspondingly $[33,35]$. The addition of the organic solvents can further reduce the water/salt ratio as well as the water activity. As a result, the cathodic limit is further expanded to $\sim 1.0 \mathrm{~V}\left(v s . \mathrm{Li}^{+} / \mathrm{Li}\right)$ and $0.7 \mathrm{~V}\left(v s . \mathrm{Li}^{+} / \mathrm{Li}\right)$. "Water-inbisalt" electrolytes have also been explored by pushing the concentration to an extremely high level, from $28 \mathrm{~mol} \mathrm{~kg}^{-1}\left(21 \mathrm{~mol} \mathrm{~kg}^{-1}\right.$ LiTFSI $+7 \mathrm{~mol} \mathrm{~kg}^{-1}$ LiOTf $)$ to $63 \mathrm{~mol} \mathrm{~kg}^{-1}\left(42 \mathrm{~mol} \mathrm{~kg}{ }^{-1}\right.$ LiTFSI $+21 \mathrm{~mol} \mathrm{~kg}^{-1}$ trimethylethyl ammonium ( $\left.\left.\mathrm{Me}_{3} \mathrm{EtN}\right) \cdot \mathrm{TFSI}\right) \quad[12,36,37]$. Nevertheless, the cathodic limit still cannot be largely expanded. Even with the $63 \mathrm{~mol} \mathrm{~kg}^{-1}$ concentration, the negative ESW can be just expanded from $1.9 \mathrm{~V}\left(v s . \mathrm{Li}^{+} / \mathrm{Li}\right)$ in $21 \mathrm{~mol} \mathrm{~kg}^{-1}$ LiTFSI to $1.75 \mathrm{~V}\left(v s . \mathrm{Li}^{+} / \mathrm{Li}\right)$ [37], which is inferior to the effect of co-solvents like DMC or AN. The reason may be that the organic co-solvents could help to form an SEI more robust than that in the co-salt electrolyte. Therefore, the SEI is the key point to expand the negative limit of the aqueous electrolytes, and the hybrid SEI composed of organics and inorganics seems to be more reliable than the SEI based on pure inorganics in the aqueous system.

To fully understand the SEI, the component analysis is just the first step, and more comprehensive information is urgently needed. The proportion of each component and the exact distribution are of more significance and usually determine the properties of the SEI, such as the mechanical strength, the ion transportation capability, and the resistance. So far, comprehensive composition information on the SEI in WiSEs and the morphology and detailed distribution of the components is unclear. For the formation mechanisms, no common understanding has yet been reached. Even so, we can still acquire some common comprehension of the SEI formation in WiSEs from previous insightful studies.

(1) The formation of the SEI in WiSEs involves multiple complicated reactions, the reactants of which may come from the salt solute, $\mathrm{H}_{2} \mathrm{O}$, and the dissolved $\mathrm{O}_{2}$ and $\mathrm{CO}_{2}$. Owing to the different thermodynamic equilibriums of the reactions, they are triggered at different potentials when the anode is negatively polarized, resulting in the heterogeneous solid deposition. Generally, the effective formation reactions should have the priority over the undesired reactions such as HER, otherwise the overwhelming HER will inhibit any deposition of the solids, leading to quick failure of the electrolytes.

(2) The reactions cannot occur independently in an ideal sequence. They interact with each other as they occur closely on both the potential scale and spatial scale. The $\mathrm{OH}^{-}$ions generated in the HER may chemically react with TFSI $^{-}$anions through nucleophilic attack, generating solid products that can deposit on the anode surface [27]. Meanwhile, the hydrogen generated in the HER would prevent the deposition of the solid products and break the passive layer, which is detrimental to the SEI formation [16].

(3) The solid products could be dissolved in the aqueous solution. As water possesses a high dielectric constant, even LiF, which has the lowest solubility $(<0.01 \mathrm{~g}$ in water), can still be dissolved in the electrolyte, especially in consideration of its in-situ nanometric formation. Thus, the newly formed solid products can dissolve into the solution instead of adhering to the surface of the electrode. To form a reliable SEI, the generation of the solid products must be faster than the dissolution. Furthermore, there must be enough solids deposited on the anode surface to achieve effective passivation, which takes a certain amount of time during the formation process. As shown in Fig. 3a, b, the open circuit voltage (OCV) can stabilize for the cells either cycled at a low rate $(0.1 \mathrm{C})$ or after extended cycling at $1 \mathrm{C}$, while for the cells, after 

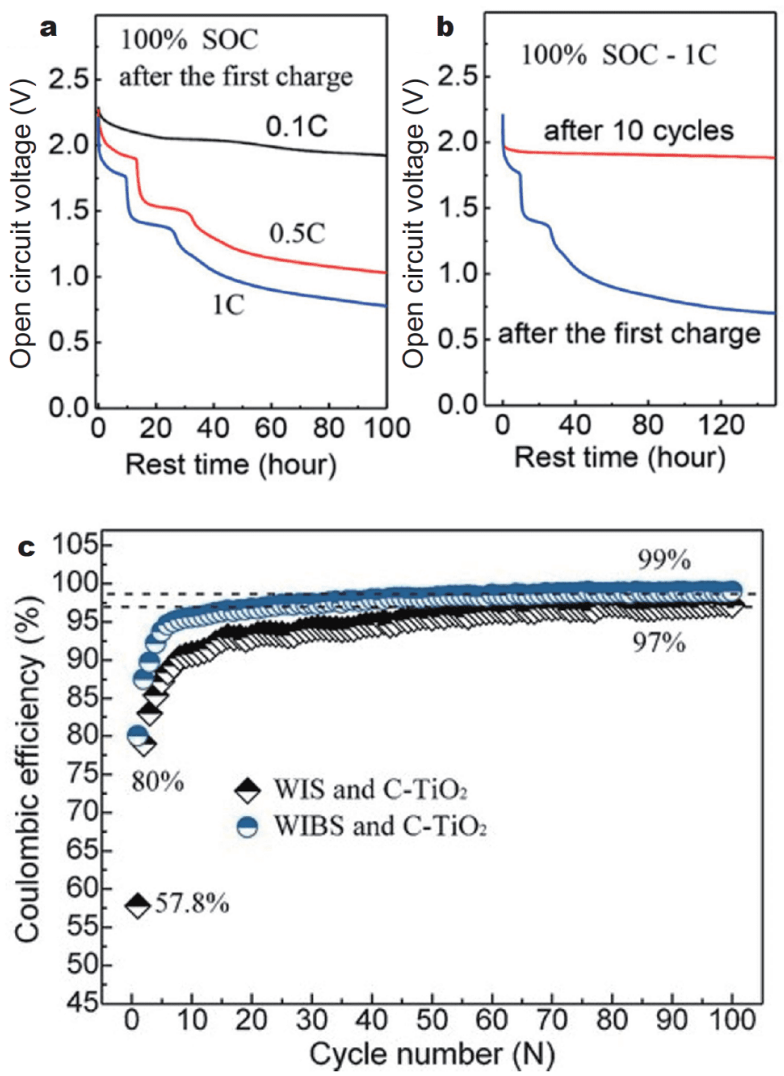

Figure 3 OCV potentials decay with rest time. (a) The cell is first fully charged to $2.3 \mathrm{~V}$ at different rates $(0.1,0.5$, and $1 \mathrm{C})$ and then rests at $100 \%$ SOC; (b) the cells are rested at $100 \%$ SOC after different cycles at a high rate (1 C). Reproduced with permission from Ref. [16], Copyright 2017, American Chemical Society. (c) The Coulombic efficiencies for varying combinations of $\mathrm{TiO}_{2}$ anode and electrolyte. Reproduced with permission from Ref. [12], Copyright 2016, WILEY-VCH Verlag GmbH \& Co. KGaA, Weinheim.

faster charging or limited cycle numbers, the OCV drops rapidly [16]. Correspondingly, the Coulombic efficiency of the full cell is relatively low in the initial several cycles, as shown in Fig. 3c [12,38]. Therefore, to effectively form the SEI and suppress the side reaction, during the initial several cycles, the anode should be polarized at specific potentials for enough time.

(4) The SEI in the aqueous system undergoes continuous dissolution and repairing growth, leading to electrolyte consumption, low Coulombic efficiency, and significantly shortened lifespan. The SEI formation routes are roughly illustrated in Fig. 4. Compared with the organic system, the high solubility may pose the biggest challenge for the in-situ formed SEI in the aqueous system. In this regard, the WiSEs not only make the SEI formation possible but also help repair and maintenance

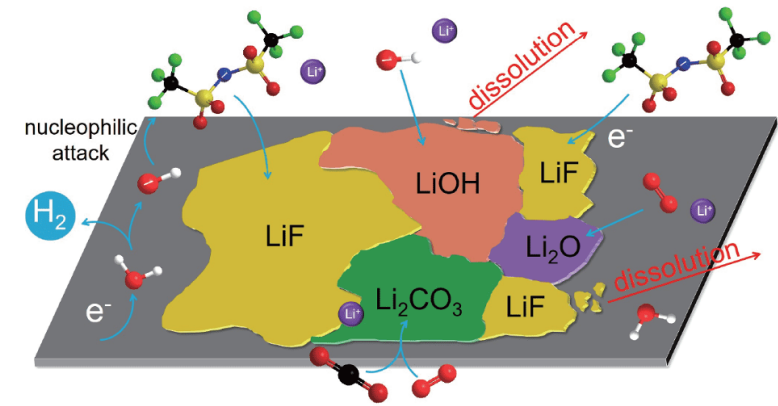

Figure 4 Schematic illustration of SEI formation process in WiSEs.

due to their adequate salt source and reduced free water ratio.

\section{FUTURE NEEDS AND PROSPECTS}

\section{Further understanding of SEI in WiSEs}

Since the discovery of the SEI in 1970 [8], researchers have devoted persistent effort to building an SEI model in the non-aqueous system in terms of the composition, the distribution, the physical/chemical properties, and the formation mechanisms. However, after nearly half a century's endeavors, the SEI is still regarded as "the most important but least understood (component) in rechargeable LIBs" due to the complex formation process and insufficient in-situ characterization tools [7,39]. Challenges can be more severe in attempts to understand the SEI in WiSEs, owing to the much harsher condition for the formation and maintenance of the SEI in the aqueous system. Even so, knowledge of the aqueous SEI will no doubt help to accelerate the development of WiSEs.

Firstly, compositional and structural information of the aqueous SEI should be clearly acquired, from which the physical/chemical properties can be inferred. The typical mosaic structure model of the SEI in non-aqueous electrolytes [9] could be referred to in order to describe the SEI in WiSEs as well, as they are both multicomponent mixtures. In view of the nanometric presence of the SEI and the high sensitivity to the ambient environment, advanced in-situ no-damage detection tools with high sensitivity and precision are urgently needed to identify the SEI components as well as their distributions. Information can also be gathered from the recent studies on non-aqueous SEIs, in which powerful characterization tools and ingenious experiment designs were applied. The tools include tip-enhanced Raman spectroscopy (TERS) [40], in-situ liquid secondary ion mass spectrometry (li- 
quid-SIMS) [41], dynamic nuclear polarization (DNP), and enhanced NMR [42]. These characterizations can not only offer compositional and structural information but also provide real-time pictures of the SEI formation. Quantum chemistry (QC) and molecular dynamics (MD) simulations are of great significance in depicting the picosecond timescale reactions at the electrode/electrolyte interface, which is rather challenging for direct experimental observation. Computational modeling can be integrated with experiments to complement each other and lead to comprehensive understanding of the SEI as well as the battery performance [39].

Quantitative investigations are encouraged, including the proportion of each component of the SEI, the reactants, and, if possible, the SEI formation process. For example, $\mathrm{Li}_{2} \mathrm{CO}_{3}$ and $\mathrm{Li}_{2} \mathrm{O}$ in the $\mathrm{SEI}$ are always attributed to the dissolved $\mathrm{CO}_{2}$ and $\mathrm{O}_{2}$, which are actually uncertain and non-quantitative as ALIBs are usually assembled in an ambient environment. Quantitative measurements of the reactive gases, the productive solids, and the reaction rate would offer some important insights into the formation process. Noticeably, the mystery of $\mathrm{O}_{2}$ is also unresolved. Luo et al. [43] have demonstrated that ALIBs cannot work sustainably in the presence of $\mathrm{O}_{2}$ because theoretically all anode materials at different reduction states would be chemically oxidized by $\mathrm{O}_{2}$ rather than undergoing the electrochemical redox process. The previous results also propose that $\mathrm{O}_{2}$ participates in the formation of the SEI in aqueous conditions. The contradiction may be explained by the quantity of $\mathrm{O}_{2}$ dissolved in the electrolytes, which calls for more elaborate experiments and quantitative evidence.

\section{Constructing more stable SEI in WiSEs}

In order to construct a robust SEI in WiSEs, the undesired side reactions such as the HER and current collector corrosion should be inhibited to make sure the SEI formation reactions dominate the interface change. Preprocessing of the anodes is expected especially when the anode material may catalyze the HER. The most popular method is coating a nanometric carbon layer on the anode material $[12,26]$, yet simpler and more effective ways for electrode pretreatment are still expected. It is important to carefully choose current collectors depending on the electrolytes and working potential and, if necessary, to passivate the current collector to prevent corrosion $[44,45]$.

There is also doubt as to what an ideal SEI in WiSEs should be. In consideration of the aqueous condition, in addition to the necessary electron insulation and ionic conduction capabilities, other properties, including high thermodynamic stability, low solubility, good adhesion, and suitable mechanical strength, are also required to maintain generation and persistence in aqueous conditions. Correlations between the interphase components and the physical/chemical properties of the SEI must be investigated. Understanding the correlations can start with investigating each specific component in its pure state, which would provide the basic criteria to evaluate an SEI and help to select the desired components. After all, the SEI is not a simple combination of each ingredient; the morphology and distribution of diverse chemical components might be as important as the components themselves. So far, the main methods used to enhance the SEI include increasing the salt concentration and introducing additional components in the SEI via adding organic co-solvents, for example, DMC, AN, and urea $[33,35,46,47]$. In consideration of the electrolyte additives, which have been extensively investigated in the organic system, additives for WiSEs can also be developed to manipulate the SEI in the aqueous system. The in-vitro artificial SEI coated on an anode may further expand the cathodic limit and enable anode materials with more negative potential to work in the WiSEs. Experience can be learned from the organic system; however, it should be noticed that the electrolyte additives and the artificial SEI in the organic system cannot be used directly in the aqueous system. The working potential of the electrodes and the stability of the SEI in aqueous system should be taken into account when developing additives and artificial SEIs in WiSE systems.

The stability of the SEI manipulated by electrolyte optimization or artificial methods needs to be roundly assessed through ESW testing as well as practical cells. Different testing conditions usually lead to inconsistent results of the ESW of certain electrolytes [26,48]. Moreover, a widened ESW tested does not always enable a high voltage output of the practical cell. Therefore, it is important to choose an appropriate working electrode and the criteria of cut-off current density when testing the ESW of the electrolytes. Furthermore, various factors need to be tested and evaluated in full cells, including the sustainability upon long-term cycling, the stability under practical operating conditions, and the compatibility with various electrode materials.

\section{SUMMARY}

It is beyond doubt that the SEI plays the key role in expanding the negative limit of the ESW for WiSEs, yet the current understanding of the SEI is just the tip of the 
iceberg. Difficulties of accurately understanding the SEI mainly come from the complicated formation mechanisms and insufficient characterization tools. More efforts should be devoted to investigating the components of the SEI as well as the distributions. Operando characterization tools and computational simulations should be developed to identify the SEI formation route. With the increasing safety concern over batteries, aqueous batteries with intrinsic nonflammable electrolytes will attract more and more attention. A comprehensive understanding of the aqueous SEI would guide the design of a more robust passive layer and further improve the electrochemical performance of ALIBs, offering the potential to compete with the state-of-art organic LIBs.

\section{Received 19 October 2020; accepted 25 December 2020;} published online 5 March 2021

1 Chao D, Zhou W, Xie F, et al. Roadmap for advanced aqueous batteries: From design of materials to applications. Sci Adv, 2020, 6: eaba4098

2 Liang T, Hou R, Dou Q, et al. The applications of water-in-salt electrolytes in electrochemical energy storage devices. Adv Funct Mater, 2021, 31: 2006749

3 Li W, Dahn JR, Wainwright DS. Rechargeable lithium batteries with aqueous electrolytes. Science, 1994, 264: 1115-1118

4 Bin D, Wen Y, Wang Y, et al. The development in aqueous lithium-ion batteries. J Energy Chem, 2018, 27: 1521-1535

5 Kim H, Hong J, Park KY, et al. Aqueous rechargeable Li and $\mathrm{Na}$ ion batteries. Chem Rev, 2014, 114: 11788-11827

$6 \mathrm{Xu} \mathrm{K}$. Nonaqueous liquid electrolytes for lithium-based rechargeable batteries. Chem Rev, 2004, 104: 4303-4418

$7 \mathrm{Xu} \mathrm{K}$. Electrolytes and interphases in Li-ion batteries and beyond. Chem Rev, 2014, 114: 11503-11618

8 Dey AN, Sullivan BP. The electrochemical decomposition of propylene carbonate on graphite. J Electrochem Soc, 1970, 117: 222224

9 Peled E. The electrochemical behavior of alkali and alkaline earth metals in nonaqueous battery systems-the solid electrolyte interphase model. J Electrochem Soc, 1979, 126: 2047-2051

10 Suo L, Borodin O, Gao T, et al. "Water-in-salt" electrolyte enables high-voltage aqueous lithium-ion chemistries. Science, 2015, 350: 938-943

11 Yamada Y, Usui K, Sodeyama K, et al. Hydrate-melt electrolytes for high-energy-density aqueous batteries. Nat Energy, 2016, 1: 16129

12 Suo L, Borodin O, Sun W, et al. Advanced high-voltage aqueous lithium-ion battery enabled by "water-in-bisalt" electrolyte. Angew Chem Int Ed, 2016, 55: 7136-7141

13 Forero-Saboya J, Hosseini-Bab-Anari E, Abdelhamid ME, et al. Water-in-bisalt electrolyte with record salt concentration and widened electrochemical stability window. J Phys Chem Lett, 2019, 10: 4942-4946

14 Li M, Wang C, Chen Z, et al. New concepts in electrolytes. Chem Rev, 2020, 120: 6783-6819

15 Borodin O, Self J, Persson KA, et al. Uncharted waters: Superconcentrated electrolytes. Joule, 2020, 4: 69-100
16 Suo L, Oh D, Lin Y, et al. How solid-electrolyte interphase forms in aqueous electrolytes. J Am Chem Soc, 2017, 139: 18670-18680

17 Yang C, Suo L, Borodin O, et al. Unique aqueous Li-ion/sulfur chemistry with high energy density and reversibility. Proc Natl Acad Sci USA, 2017, 114: 6197-6202

18 Suo L, Borodin O, Wang Y, et al. "Water-in-salt" electrolyte makes aqueous sodium-ion battery safe, green, and long-lasting. Adv Energy Mater, 2017, 7: 1701189

19 Leonard DP, Wei Z, Chen G, et al. Water-in-salt electrolyte for potassium-ion batteries. ACS Energy Lett, 2018, 3: 373-374

20 Wang F, Fan X, Gao T, et al. High-voltage aqueous magnesium ion batteries. ACS Cent Sci, 2017, 3: 1121-1128

21 Dou Q, Lei S, Wang DW, et al. Safe and high-rate supercapacitors based on an "acetonitrile/water in salt" hybrid electrolyte. Energy Environ Sci, 2018, 11: 3212-3219

$22 \mathrm{Bu}$ X, Su L, Dou Q, et al. A low-cost "water-in-salt" electrolyte for a $2.3 \mathrm{~V}$ high-rate carbon-based supercapacitor. J Mater Chem A, 2019, 7: 7541-7547

23 Yang C, Chen J, Qing T, et al. $4.0 \mathrm{~V}$ aqueous Li-ion batteries. Joule, 2017, 1: 122-132

24 Vatamanu J, Borodin O. Ramifications of water-in-salt interfacial structure at charged electrodes for electrolyte electrochemical stability. J Phys Chem Lett, 2017, 8: 4362-4367

25 Zheng J, Tan G, Shan P, et al. Understanding thermodynamic and kinetic contributions in expanding the stability window of aqueous electrolytes. Chem, 2018, 4: 2872-2882

26 Lukatskaya MR, Feldblyum JI, Mackanic DG, et al. Concentrated mixed cation acetate "water-in-salt" solutions as green and lowcost high voltage electrolytes for aqueous batteries. Energy Environ Sci, 2018, 11: 2876-2883

27 Dubouis N, Lemaire P, Mirvaux B, et al. The role of the hydrogen evolution reaction in the solid-electrolyte interphase formation mechanism for "water-in-salt" electrolytes. Energy Environ Sci, 2018, 11: 3491-3499

28 Lee MH, Kim SJ, Chang D, et al. Toward a low-cost high-voltage sodium aqueous rechargeable battery. Mater Today, 2019, 29: 2636

29 Zhang SS. A review on electrolyte additives for lithium-ion batteries. J Power Sources, 2006, 162: 1379-1394

30 Wang C, Meng YS, Xu K. Perspective-fluorinating interphases. J Electrochem Soc, 2019, 166: A5184-A5186

31 Pan J, Cheng YT, Qi Y. General method to predict voltagedependent ionic conduction in a solid electrolyte coating on electrodes. Phys Rev B, 2015, 91: 134116

32 Zhang Q, Pan J, Lu P, et al. Synergetic effects of inorganic components in solid electrolyte interphase on high cycle efficiency of lithium ion batteries. Nano Lett, 2016, 16: 2011-2016

33 Wang F, Borodin O, Ding MS, et al. Hybrid aqueous/non-aqueous electrolyte for safe and high-energy Li-ion batteries. Joule, 2018, 2 : 927-937

34 Zhang H, Qin B, Han J, et al. Aqueous/nonaqueous hybrid electrolyte for sodium-ion batteries. ACS Energy Lett, 2018, 3: 17691770

35 Chen J, Vatamanu J, Xing L, et al. Improving electrochemical stability and low-temperature performance with water/acetonitrile hybrid electrolytes. Adv Energy Mater, 2019, 10: 1902654

36 Jiang $\mathrm{P}$, Chen L, Shao H, et al. Methylsulfonylmethane-based deep eutectic solvent as a new type of green electrolyte for a highenergy-density aqueous lithium-ion battery. ACS Energy Lett, 2019, 4: 1419-1426 
37 Chen L, Zhang J, Li Q, et al. A $63 \mathrm{~m}$ superconcentrated aqueous electrolyte for high-energy Li-ion batteries. ACS Energy Lett, 2020, 5: $968-974$

38 Suo L, Han F, Fan X, et al. "Water-in-salt" electrolytes enable green and safe Li-ion batteries for large scale electric energy storage applications. J Mater Chem A, 2016, 4: 6639-6644

39 Wang A, Kadam S, Li H, et al. Review on modeling of the anode solid electrolyte interphase (SEI) for lithium-ion batteries. npj Comput Mater, 2018, 4: 15

40 Nanda J, Yang G, Hou T, et al. Unraveling the nanoscale heterogeneity of solid electrolyte interphase using tip-enhanced Raman spectroscopy. Joule, 2019, 3: 2001-2019

41 Zhou Y, Su M, Yu X, et al. Real-time mass spectrometric characterization of the solid-electrolyte interphase of a lithium-ion battery. Nat Nanotechnol, 2020, 15: 224-230

42 Hope MA, Rinkel BLD, Gunnarsdóttir AB, et al. Selective NMR observation of the SEI-metal interface by dynamic nuclear polarisation from lithium metal. Nat Commun, 2020, 11: 2224

43 Luo JY, Cui WJ, He P, et al. Raising the cycling stability of aqueous lithium-ion batteries by eliminating oxygen in the electrolyte. Nat Chem, 2010, 2: 760-765

44 Kühnel RS, Reber D, Remhof A, et al. "Water-in-salt" electrolytes enable the use of cost-effective aluminum current collectors for aqueous high-voltage batteries. Chem Commun, 2016, 52: 1043510438

45 Hou Z, Zhang X, Ao $\mathrm{H}$, et al. Passivation effect for current collectors enables high-voltage aqueous sodium ion batteries. Mater Today Energy, 2019, 14: 100337

46 Hou Z, Dong M, Xiong Y, et al. Formation of solid-electrolyte interfaces in aqueous electrolytes by altering cation-solvation shell structure. Adv Energy Mater, 2020, 10: 1903665

47 Shang Y, Chen N, Li Y, et al. An "ether-in-water" electrolyte boosts stable interfacial chemistry for aqueous lithium-ion batteries. Adv Mater, 2020, 32: 2004017

48 Kühnel RS, Reber D, Battaglia C. Perspective-electrochemical stability of water-in-salt electrolytes. J Electrochem Soc, 2020, 167: 070544

Acknowledgements This work was supported by the National Natural Science Foundation of China (22075091 and 21773077).

Author contributions Liu D collected the related literatures and wrote the manuscript with support from Yuan L. Huang Y provided critical feedback and helped shape the manuscript.

\section{Conflict of interest}

These authors declare no conflict of interest.

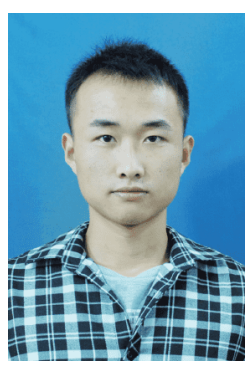

Dezhong Liu received his BSc degree from the School of Materials Science and Engineering, Huazhong University of Science and Technology (HUST), in 2016. He is currently a $\mathrm{PhD}$ candidate in the School of Materials Science and Engineering at HUST. His current research mainly focuses on the sulfur-based materials and their applications in aqueous rechargeable batteries.

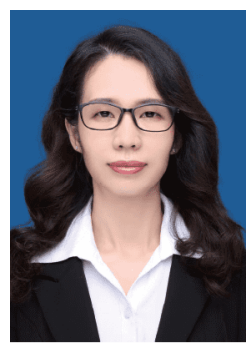

Lixia Yuan received her BSc, MSc and PhD degrees from Wuhan University. She worked as a post-doctoral researcher at Tsinghua University in 2007-2009. She is now a professor at HUST. Her research interests mainly focus on lithium rechargeable batteries.

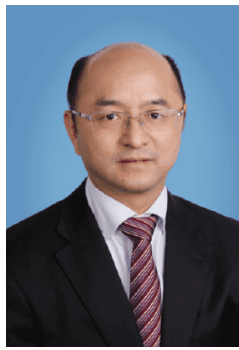

Yunhui Huang received his BSc, MSc and $\mathrm{PhD}$ degrees from Peking University. From 2002 to 2004, he worked as an associate professor at Fudan University. He then worked with Prof. John B. Goodenough in the University of Texas at Austin for more than three years. In 2008, he became a chair professor of materials science at HUST. His research group works on the rechargeable batteries and electrode materials.

\section{Water-in-salt电解液中的固态电解质界面膜}

刘德重, 袁利霞, 黄云辉

摘要 Water-in-salt (WIS) 电解液成功将水溶液电解质的电化学稳 定窗口从 $1.23 \mathrm{~V}$ 拓宽至 $3.0 \mathrm{~V}$ 以上, 在保留了水系电池高安全性的同 时, 显著提高了水系电池的输出电压和能量密度. 其电化学稳定窗 口的拓宽主要归因于高盐电解质体系中水分子的活性降低以及在 负极表面形成的固态电解质界面(SEI)膜. 目前, 对水系SEI膜的认 识尚不全面, 其基本成分、结构、形成机理仍然是一片谜团. 本文 对目前已有的研究工作进行了总结, 着重探讨了水系SEI膜最有可 能的形成机理, 并期望能为其进一步研究以及实际运行条件下WIS 电解液的全面评估提供理论基础. 深入理解WIS体系中的电极/电 解质界面, 对于水性电解液的设计以及高安全、高能量密度的新 型水系电池的构筑均具有重要意义. 